\title{
Minimax Robust Decentralized Detection
}

\author{
Venugopal V. Veeravalli, Member, IEEE, Tamer Başar, Fellow, IEEE, \\ and $\mathrm{H}$. Vincent Poor, Fellow, IEEE
}

\begin{abstract}
Decentralized detection problems are studied where the sensor distributions are not specified completely. The sensor distributions are assumed to belong to known uncertainty classes. It is shown for a broad class of such problems that a set of least favorable distributions exists for minimax robust testing between the hypotheses. It is hence established that the corresponding minimax robust tests are solutions to simple decentralized detection problems for which the sensor distributions are specified to be the least favorable distributions.
\end{abstract}

Index Terms-Decentralized detection, robust hypothesis testing, least favorable distributions, minimax optimization.

\section{INTRODUCTION}

$\mathbf{T}$ HE design of optimal decision rules in detection (hypothesis testing) problems requires the knowledge of the conditional probability distributions of the observations, given each hypothesis. In many applications, however, the probability distributions are not specified completely. In these cases, the probability distributions are usually specified to belong to classes (sets) of distributions, often termed uncertainty classes. One way to design decision rules when the probability distributions are given to belong to uncertainty classes is the minimax approach, where the goal is to minimize the worst case performance over the uncertainty classes. The decision rules thus obtained are said to be robust to the uncertainties in the probability distributions.

Minimax robust detection problems with two hypotheses ${ }^{1}$ and with centralized information have been the subject of numerous papers (for a survey of results in this area, see [2]). The solutions to these problems invariably involve identifying a pair of least favorable distributions (LFD's), and subsequently designing a simple hypothesis test between the the LFD's.

With the increasing interest in decentralized detection in recent years, extensions of centralized detection problems to their corresponding decentralized formulations have been studied (see [3] for a survey of recent results). An extension

Manuscript received July 29, 1992; revised May 15, 1993. This work was supported in part by the Joint Services Electronics Program under Grant N00 014-90-J-1270 through the University of Illinois. This paper was presented in part at the 26th Annual Conference on Information Sciences and Systems, Princeton, NJ, Mar. 1992.

V. V. Veeravalli was with the Department of Electrical and Computer Engineering and the Coordinated Science Laboratory, University of Illinois, Urbana, IL 61801. He is now with the Department of Electrical Engineering, City University of New York, New York, NY 10031.

T. Başar is with the Department of Electrical and Computer Engineering, and the Coordinated Science Laboratory, University of Illinois, Urbana, IL 61801.

H. V. Poor is with the Department of Electrical Engineering, Princeton University, Princeton, NJ 08544.

IEEE Log Number 9215120.

${ }^{1}$ Minimax robust detection problems with more than two hypotheses are known to be difficult and do not admit closed-form solutions [1]. of the minimax robust detection problem to a decentralized setting with two sensors and without a fusion center was considered by Geraniotis [4]. The problem was formulated in a Bayesian framework with the observations at each of the sensors belonging to uncertainty classes generated by alternating capacities of order two. The binary sensor decisions about the hypothesis were assumed to be coupled through a common cost function. For a specific choice of cost structure, it was shown in [4] that the task of finding LFD's at the sensors can be decoupled into two independent tasks, one at each of the sensors. This implies that the LFD's for the decentralized problem are the same as those for two independent centralized detection problems at the sensors.

Minimax robust decentralized detection with a fusion center has also been studied. In the only existing analyses of this problem [5], [6], the authors restricted their study to a Bayesian formulation and to binary sensor decisions. They further limited the scope of their study by only considering the following special cases: 1) the case of identical sensors using identical decision rules, 2) the asymptotic case of a large number of sensors, and 3) the asymptotic case of large observation block lengths.

In this paper, we attempt to find a more comprehensive solution to robust decentralized detection problems. We study both cases with and without a fusion center. For the case when a fusion center is present, we give a solution to the minimax robust detection problem for the general case of finite number of sensors, finite observation block length, and nonbinary sensor decisions. This solution covers all the block detection cases considered in [5] and [6]. Furthermore, our analysis is not restricted to Bayesian detection. For the case when no fusion center is present, we extend the work in [4] to more than two sensors and more general cost functions. We also give sufficient conditions for the decoupling of the minimax robust detection problem.

The remainder of this paper is organized as follows. In Section II, we give a detailed introduction to robust centralized detection. The purpose of this Introduction is twofold: first, we believe that we have provided a framework whereby most of the previous results in robust centralized detection are unified; second, the results here are used explicitly in the solution to the decentralized problems in the subsequent sections. In Section III, we consider decentralized detection problems where a fusion center is present, and in Section IV, we consider the case where the fusion center is absent. Finally, in Section V, we give some concluding remarks. An Appendix containing the proof of one of the results is included at the end of the paper. 


\section{RoBUST CENTRALIZED DETECTION}

We begin with a description of a minimax robust detection problem which was first introduced by Huber [7]. The basic setup is as follows. Let $(\mathcal{X}, \mathcal{F})$ be a measurable space, and let $P_{0}$ and $P_{1}$ be distinct probability measures on it. Let $X$ be an observation taking values in $\mathcal{X}$, and let the distribution of $X$ be $P_{0}$ (respectively, $P_{1}$ ) under $H_{0}$ (respectively, $H_{1}$ ). A decision $\delta$ about the true hypothesis is to be made based on $X$, i.e., $\delta=\phi(X)$. The objective here is to construct a hypothesis test between $H_{0}$ and $H_{1}$, when $P_{0}$ and $P_{1}$ are not specified completely. The approach taken by Huber was to first define classes of allowable distributions (or uncertainty classes) under $H_{0}$ and $H_{1}$, and then solve a minimax test between this pair of classes. If we denote the uncertainty class under $H_{j}$ by $\mathcal{P}_{j}$, then the minimax robust versions of Bayesian, minimax, and Neyman-Pearson formulations of the hypothesis test between $H_{0}$ and $H_{1}$ are given, respectively, by

a) $\min _{\phi}\left[\nu \sup _{P_{0} \in \mathcal{P}_{0}} P_{F}\left(\phi, P_{0}\right)+(1-\nu) \sup _{P_{1} \in \mathcal{P}_{1}} P_{M}\left(\phi, P_{1}\right)\right]$

b) $\min _{\phi} \max \left\{\sup _{P_{0} \in \mathcal{P}_{0}} P_{F}\left(\phi, P_{0}\right), \sup _{P_{1} \in \mathcal{P}_{1}} P_{M}\left(\phi, P_{1}\right)\right\}$

c) $\min _{\phi} \sup _{P_{1} \in \mathcal{P}_{1}} P_{M}\left(\phi, P_{1}\right)$

$$
\text { subject to } \sup _{P_{0} \in \mathcal{P}_{0}} P_{F}\left(\phi, P_{0}\right) \leq \alpha
$$

where $P_{M}\left(\phi, \mathcal{P}_{1}\right)=P_{1}(\delta=0)$ and $P_{F}\left(\phi, \mathcal{P}_{0}\right)=P_{0}(\delta=1)$, and $\nu$ is the a priori probability of hypothesis $H_{0}$.

The classes considered in [7] are neighborhood classes containing, under each hypothesis, a nominal distribution and distributions in its vicinity. The two types of neighborhood classes studied in [7] are the $\epsilon$ contamination and the total variation. For each case, Huber showed [7] that a pair of LFD's can be found for the minimax robust detection problems described above. He also gave a characterization of a least favorable pair in terms of the parameters of the uncertainty neighborhoods, and showed that the corresponding minimax robust tests are "censored" versions of the nominal likelihood ratio tests.

Huber and Strassen [8] have shown in a later paper that pairs of LFD's can be found for the cases when the neighborhood classes can be described in terms of alternating capacities of order 2 . When the observation set is compact, several uncertainty models such as $\epsilon$-contamination neighborhoods, total variation neighborhoods, band classes, and $p$-point classes are special cases of this model with different choices of capacity.

The proofs of existence of LFD's in [7] and [8] rely on the following property possessed by all the pairs of uncertainty classes considered in [7] and [8].

Definition 1 (Joint Stochastic Boundedness): A pair $\left(\mathcal{P}_{0}\right.$, $\mathcal{P}_{1}$ ) of classes of distributions defined on a measurable space $(\mathcal{X}, \mathcal{F})$ is said to be jointly stochastically bounded by $\left(Q_{0}, Q_{1}\right)$, if there exist distributions $Q_{0} \in \mathcal{P}_{0}$ and $Q_{1} \in \mathcal{P}_{1}$ such that, for any $\left(P_{0}, P_{1}\right) \in \mathcal{P}_{0} \times \mathcal{P}_{1}$ and all $t \geq 0$,

$$
P_{0}\left(l_{q}(X)>t\right) \leq Q_{0}\left(l_{q}(X)>t\right)
$$

and

$$
P_{1}\left(l_{q}(X)>t\right) \geq Q_{1}\left(l_{q}(X)>t\right)
$$

where $l_{q}$ is the likelihood ratio between $Q_{1}$ and $Q_{0}$.
It can be shown [7], [8] that the distributions $Q_{0}$ and $Q_{1}$ in Definition 1 are LFD's for minimax robust hypothesis testing between $\mathcal{P}_{0}$ and $\mathcal{P}_{1}$. That is, the solution to a), b), and $c$ ) are obtained as solutions to the following simple hypothesis testing problems:

$\left.\mathrm{a}^{\prime}\right) \min _{\phi}\left[\nu P_{F}\left(\phi, Q_{0}\right)+(1-\nu) P_{M}\left(\phi, Q_{1}\right)\right]$

$\left.\mathrm{b}^{\prime}\right) \min _{\phi} \max \left\{\left(P_{F}\left(\phi, Q_{0}\right), P_{M}\left(\phi, Q_{1}\right)\right\}\right.$

c') $\min _{\phi} P_{M}\left(\phi, Q_{1}\right)$ subject to $P_{F}\left(\phi, Q_{0}\right) \leq \alpha$.

In many applications, the observation $X$ is a vector (block) of independent observations $\left(X_{1}, \cdots, X_{n}\right)$, where the observation $X_{i}$ takes values in a measurable space $\left(\mathcal{X}_{i}, \mathcal{F}_{i}\right)$ and has a distribution which belongs to the class $\mathcal{P}_{j}^{i}$ when the hypothesis is $H_{j}$. In this paper, the set $\mathcal{P}_{j}:=\mathcal{P}_{j}^{1} \times \cdots \times \mathcal{P}_{j}^{n}$ represents a class of distributions on $(\mathcal{X}, \mathcal{F})$ which are products of distributions in $\mathcal{P}_{j}^{i}, i=1, \cdots n$. To further clarify this point, let $P_{j}^{i}$ denote a typical element of $\mathcal{P}_{j}^{i}$. The $P_{j}:=\left(P_{j}^{1}, \cdots, P_{j}^{n}\right) \in$ $\mathcal{P}_{j}$ represents the product distribution $P_{j}^{1} \times \cdots \times P_{j}^{n}$.

In the above context, we have the following result.

Lemma 1: For each $i, i=1, \cdots, n$, let the pair $\left(\mathcal{P}_{0}^{i}, \mathcal{P}_{1}^{i}\right)$ be jointly stochastically bounded by $\left(Q_{0}^{i}, Q_{1}^{i}\right)$. Then the pair $\left(\mathcal{P}_{0}, \mathcal{P}_{1}\right)$ is jointly stochastically bounded by $\left(Q_{0}, Q_{1}\right)$.

The proof of Lemma 1 follows quite easily from the following result which is proved in the Appendix.

Lemma 2: Let $Z_{1}, Z_{2}, \cdots, Z_{n}$ be nonnegative, independent random variables. Let $Z_{i}$ have distribution $\mathcal{F}_{i}$ under measure $\mathcal{F}$, and have distribution $\mathcal{G}_{i}$ under measure $\mathcal{G}$. Furthermore, suppose that $Z_{i}$ is stochastically larger under $\mathcal{F}_{i}$ than under $\mathcal{G}_{i}$, i.e.,

$$
\mathcal{F}_{i}\left(Z_{i}>t_{i}\right) \geq \mathcal{G}_{i}\left(Z_{i}>t_{i}\right), \quad \text { for all } t_{i} \geq 0 .
$$

Then $\prod_{i=1}^{n} Z_{i}$ is stochastically larger under $\mathcal{F}$ than under $\mathcal{G}$, i.e.,

$$
\mathcal{F}\left(\prod_{i=1}^{n} Z_{i}>t\right) \geq \mathcal{G}\left(\prod_{i=1}^{n} Z_{i}>t\right) \quad \text { for all } t \geq 0 .
$$

Proof of Lemma 1: Let $P_{0}$ be any distribution in the set $\mathcal{P}_{0}$, and let $P_{1}$ be any distribution in the set $\mathcal{P}_{1}$. Let $l_{q}$ denote the likelihood ratio between $Q_{1}$ and $Q_{0}$, and let $l_{q}^{i}$ denote the likelihood ratio between $Q_{1}^{i}$ and $Q_{0}^{i}$. Then

$$
l_{q}(X)=\prod_{i=1}^{n} l_{q}^{i}\left(X_{i}\right)
$$

By the joint stochastic boundedness property of $\left(\mathcal{P}_{0}^{1}, \mathcal{P}_{1}^{i}\right)$, $l_{1}^{i}\left(X_{i}\right)$ is stochastically larger under $Q_{0}^{i}$ than under $P_{0}^{i}$. Hence, by Lemma $2, l_{q}(X)$ is stochastically larger under $Q_{0}$ than under $P_{0}$. This proves the first condition required for the joint stochastic boundedness of $\left(\mathcal{P}_{0}, \mathcal{P}_{1}\right)$ by $\left(Q_{0}, Q_{1}\right)$. The other condition is proved similarly.

Remark 1: Huber [7] proved Lemma 1 for the special case when when $\mathcal{P}_{j}^{i}=\mathcal{P}_{j}^{1}$, for $i=2, \cdots, n$. We note that the proof given here is a straightforward extension of Huber's proof. Also, even for the case when $\mathcal{P}_{j}^{i}=\mathcal{P}_{j}^{1}$, for $i=2, \cdots, n$, the individual members of $\mathcal{P}_{j}$ need not be vectors of identical distributions. That is, Huber's result, as given in [7], applies to more than just independent and identically distributed observations; Lemma 1 is a further generalization of this result. 


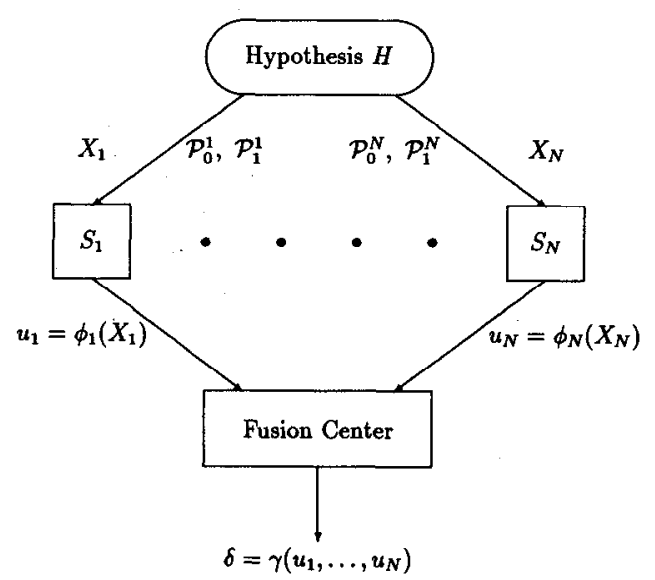

Fig. 1. Robust decentralized detection with a fusion center

Lemma 1 implies the following. Suppose we are given a minimax robust detection problem with a block of independent observations, and with uncertainty classes satisfying the joint stochastic boundedness property. Then, this problem can be reduced to a single observation problem without sacrificing the joint stochastic boundedness property.

The above description of centralized robust detection problems, we believe, unifies most of the results in this area. Hence, the material of Section II should be of independent interest to the reader.

\section{RoBUST DECENTRALIZED}

\section{DETECTION WITH A FUSION CENTER}

A description of the decentralized detection system considered in this section is given in Fig. 1. The hypothesis $H$ takes values $H_{0}$ and $H_{1}$. There are $N$ sensors and one fusion center. The sensor $S_{i}$ receives an observation $X_{i}$ which is assumed to take values on a measurable space $\left(\mathcal{X}_{i}, \mathcal{F}_{i}\right)$. By virtue of Lemma $1, X_{i}$ could represent a block of independent observations. The observations at the sensors are independent, and $X_{i}$ has a distribution which belongs to the class $\mathcal{P}_{j}^{i}$ when the hypothesis is $H_{j}$. For each $i$, the pair of uncertainty classes $\left(P_{0}^{i}, \mathcal{P}_{1}^{i}\right)$ is jointly stochastically bounded by $\left(Q_{0}^{i}, Q_{1}^{i}\right)$ (see Definition 1). Let $\mathcal{P}_{j}:=\mathcal{P}_{j}^{1} \times \cdots \times \mathcal{P}_{j}^{N}$. Then $P_{j}=\left(P_{j}^{1}, \cdots, P_{j}^{N}\right) \in \mathcal{P}_{j}$ represents the product distribution $P_{j}^{1} \times, \cdots, \times P_{j}^{N}$.

By Lemma 1 , the pair $\left(\mathcal{P}_{0}, \mathcal{P}_{1}\right)$ is jointly stochastically bounded by $\left(Q_{0}, Q_{1}\right)$. Hence, if all of the information received by the sensors is made available to the fusion center, then $\left(Q_{0}, Q_{1}\right)$ are LFD's for robust hypothesis testing between $H_{0}$ and $H_{1}$. But in the decentralized setting, only a summary of the sensor observations is available al the fusion center. At sensor $S_{i}$, there is a decision function $\phi_{i}$ which maps the observation vector $X_{i}$ to a local decision $U_{i} \in\left\{1, \cdots, D_{i}\right\}$, and the fusion center makes a final binary-valued decision $\delta$ based on the information it receives from the sensors, i.e., $\delta=\gamma\left(U_{1}, \cdots, U_{N}\right)$. For compactness of notation, we represent the set of local decision functions $\left(\phi_{1}, \cdots, \phi_{N}\right)$ by $\phi$.

We consider here, in detail, a Bayesian formulation of the robust decentralized detection problem where the objective is to minimize the worst case error probability at the fusion center. The minimax and Neyman-Pearson formulations are discussed briefly at the end of this section.

The hypothesis is assumed to take on values $H_{0}$ and $H_{1}$, with prior probabilities $\nu$ and $1-\nu$, respectively. Let $P_{F}\left(P_{0}, \phi, \gamma\right):=P_{0}(\delta=1)$ and $P_{M}\left(P_{1}, \phi, \gamma\right):=P_{1}(\delta=0)$. The the problem we wish to solve is the following.

Problem (P1):

$$
\inf _{\phi, \gamma}\left[\nu \sup _{P_{0} \in \mathcal{P}_{0}} P_{F}\left(P_{0}, \phi, \gamma\right)+(1-\nu) \sup _{P_{1} \in \mathcal{P}_{1}} P_{M}\left(P_{1}, \phi, \gamma\right)\right] \text {. }
$$

In the following, we will establish that $\left(Q_{0}, Q_{1}\right)$, which were LFD's for the centralized problem, are LFD's for Problem (P1) as well. That is, the solution to (P1) is obtained as the solution to the simple decentralized detection problem $\left(\mathrm{P}^{\prime}\right)$ given below.

\section{Problem $\left(P 1^{\prime}\right)$ :}

$$
\inf _{\phi, \gamma}\left[\nu P_{F}\left(Q_{0}, \phi, \gamma\right)+(1-\nu) P_{M}\left(Q_{1}, \phi, \gamma\right)\right]
$$

Optimal decision rules for Problem $\left(\mathrm{P}^{\prime}\right)$ are monotone likelihood ratio tests (MLRT's) [3] of the form

$$
\phi_{i}^{R}\left(X_{i}\right)= \begin{cases}1 & \text { if } l_{q}^{i}\left(X_{i}\right)<\lambda_{1}^{i} \\ d & \text { if } \lambda_{d-1}^{i} \leq l_{q}^{i}\left(X_{i}\right)<\lambda_{d}^{i} \\ & d=2, \cdots, D_{i}-1 \\ D_{i} & \text { if } l_{q}^{i}\left(X_{i}\right) \geq \lambda_{D_{i}}^{i}\end{cases}
$$

and

$$
\gamma^{R}\left(U_{1}, \cdots, U_{N}\right)= \begin{cases}1 & \text { if } l_{q}\left(U_{1}, \cdots, U_{N}\right)>t \\ 0 & \text { otherwise }\end{cases}
$$

where

$$
l_{q}\left(u_{1}, \cdots, u_{N}\right)=\prod_{i=1}^{N} \frac{Q_{1, U}^{i}\left(u_{i}\right)}{Q_{0, U}^{i}\left(u_{i}\right)}
$$

In the above expressions, $l_{q}^{i}$ denotes the generalized likelihood ratio between $Q_{1}^{i}$ and $Q_{0}^{i}$. Also, $Q_{j, U}^{i}$ denotes the probability mass function induced on $U_{i}$ by $Q_{j}^{i}$.

Remark 2: Without loss of generality, we can assume the following: a) $Q_{0, U}^{i}(d)$ is strictly positive for each $d \in\left\{1, \cdots, D_{i-1}\right\}$, and b) either $Q_{0, U}^{i}\left(D_{i}\right)$ is strictly positive or $Q_{1, U}^{i}\left(D_{i}\right) / Q_{0, U}^{i}\left(D_{i}\right)=\infty$. The reason is as follows. Suppose $Q_{0, U}^{i}(d)=0$, for some $d \in\left\{1, \cdots, D_{i-1}\right\}$ (which implies that $Q_{1, U}^{i}(d)=0$ ); then the optimal test never chooses decision $d$ at sensor $S_{i}$. This means that the decision set at sensor $S_{i}$ can be reduced by 1 (and renumbered 1 through $\left.D_{i-1}\right)$ without losing optimality. If $Q_{0, U}^{i}\left(D_{i}\right)=0$, then cither $Q_{1, U}^{i}\left(D_{i}\right)$ is zero, in which case the optimal test never chooses $D_{i}$ at $S_{i}$ and the decision set at $S_{i}$ can be reduced by 1 without losing optimality, or $Q_{1, U}^{i}\left(D_{i}\right)$ is nonzcro, in which case $Q_{1, U}^{i}\left(D_{i}\right) / Q_{0, U}^{i}\left(D_{i}\right)=\infty$.

Theorem 1: Let $\phi^{R}, \gamma^{R}$ be any set of MLRT's based on $Q_{0}$ and $Q_{1}$. Then for all $\left(P_{0}, P_{1}\right) \in \mathcal{P}_{0} \times \mathcal{P}_{1}$, we have

$$
\begin{gathered}
P_{F}\left(Q_{0}, \phi^{R}, \gamma^{R}\right) \geq P_{F}\left(P_{0}, \phi^{R}, \gamma^{R}\right), \\
P_{M}\left(Q_{1}, \phi^{R}, \gamma^{R}\right) \geq P_{M}\left(P_{1}, \phi^{R}, \gamma^{R}\right),
\end{gathered}
$$


Before we address the proof of this result, we consider its implications. Suppose $\phi^{*}, \gamma^{*}$ constitute a solution to $\left(\mathrm{P}^{\prime}\right)$. Then

$$
\begin{aligned}
\nu \sup _{P_{0} \in \mathcal{P}_{0}} & P_{F}\left(P_{0}, \phi^{*}, \gamma^{*}\right)+(1-\nu) \sup _{P_{1} \in \mathcal{P}_{1}} P_{M}\left(P_{1}, \phi^{*}, \gamma^{*}\right) \\
& =\nu P_{F}\left(Q_{0}, \phi^{*}, \gamma^{*}\right)+(1-\nu) P_{M}\left(Q_{1}, \phi^{*}, \gamma^{*}\right) \\
& \leq \nu P_{F}\left(Q_{0}, \phi, \gamma\right)+(1-\nu) P_{M}\left(Q_{1}, \phi, \gamma\right) \\
& \leq \nu \sup _{P_{0} \in \mathcal{P}_{0}} P_{F}\left(P_{0}, \phi, \gamma\right)+(1-\nu) \sup _{P_{1} \in \mathcal{P}_{1}} P_{M}\left(P_{1}, \phi, \gamma\right)
\end{aligned}
$$

for any $\phi, \gamma$. This means that $\left(Q_{0}, Q_{1}\right)$ are LFD's for (P1), and that $\phi^{*}, \gamma^{*}$ solve (P1).

We now state and prove the following lemma which will be used in the proof of Theorem 1.

Lemma 3: Let $\lambda_{1}, \lambda_{2}, \lambda_{3}, \lambda_{4}$ be such that $0 \leq \lambda_{1} \leq \lambda_{2} \leq$ $\lambda_{3} \leq \lambda_{4}<\infty$. Suppose that $Q_{0}^{i}\left(\lambda_{1} \leq l_{q}^{i}\left(X_{i}\right) \leq \lambda_{2}\right)>0$ and $Q_{0}^{i}\left(\lambda_{3} \leq l_{q}^{i}\left(X_{i}\right) \leq \lambda_{4}\right)>0$. Then

$$
\frac{Q_{1}^{i}\left(\lambda_{1} \leq l_{q}^{i}\left(X_{i}\right) \leq \lambda_{2}\right)}{Q_{0}^{i}\left(\lambda_{1} \leq l_{q}^{i}\left(X_{i}\right) \leq \lambda_{2}\right)} \leq \frac{Q_{1}^{i}\left(\lambda_{3} \leq l_{q}^{i}\left(X_{i}\right) \leq \lambda_{4}\right)}{Q_{0}^{i}\left(\lambda_{3} \leq l_{q}^{i}\left(X_{i}\right) \leq \lambda_{4}\right)}
$$

Proof: The LHS above can be written as

$$
\frac{1}{Q_{0}^{i}\left(\lambda_{1} \leq l_{q}^{i}\left(X_{i}\right) \leq \lambda_{2}\right)} \int_{\left\{\lambda_{1} \leq l_{q}^{i}(x) \leq \lambda_{2}\right\}} l_{q}^{i}(x) d Q_{0}^{i}(x) .
$$

This means that the LHS is the average of $l_{q}^{i}(x)$ with respect to the distribution $Q_{0}^{i}$ on the set $\left\{\lambda_{1} \leq l_{q}^{i}(x) \leq \lambda_{2}\right\}$, and hence is in between $\lambda_{1}$ and $\lambda_{2}$. Similarly, the RHS is between $\lambda_{3}$ and $\lambda_{4}$. The lemma follows.

Proof of Theorem 1: For any $\left(P_{0}, P_{1}\right) \in \mathcal{P}_{0} \times \mathcal{P}_{1}$,

$$
P_{F}\left(P_{0}, \phi^{R}, \gamma^{R}\right)=P_{0}\left(l_{q}\left(U_{1}, \cdots, U_{N}\right)>t\right)
$$

First, we note that

$$
l_{q}\left(U_{1}, \cdots, U_{N}\right)=\prod_{i=1}^{N} \frac{Q_{1, U}^{i}\left(U_{i}\right)}{Q_{0, U}^{i}\left(U_{i}\right)}
$$

Now, by Lemma 3 and the discussion given in Remark 2, $Q_{1, U}^{i}\left(U_{i}\right) / Q_{0, U}^{i}\left(U_{i}\right)$ is a nondecreasing function of $U_{i}$, and hence, for any $t_{i} \geq 0$,

$$
\left\{\frac{Q_{1, U}^{i}\left(U_{i}\right)}{Q_{0, U}^{i}\left(U_{i}\right)}>t_{i}\right\}=\left\{l_{q}^{i}\left(X_{i}\right)>\lambda_{t_{i}}\right\}
$$

for an appropriately chosen $\lambda_{t_{i}}$.

An application of the joint stochastic boundedness property of the pairs $\left(\mathcal{P}_{0}^{i}, \mathcal{P}_{1}^{i}\right)$ gives us that

$$
Q_{0}^{i}\left(l_{q}^{i}\left(X_{i}\right)>\lambda_{t_{i}}\right) \geq P_{0}^{i}\left(l_{q}^{i}\left(X_{i}\right)>\lambda_{t_{i}}\right) .
$$

This means that $Q_{1, U}^{i}\left(U_{i}\right) / Q_{0, U}^{i}\left(U_{i}\right)$ is stochastically larger under $Q_{0}^{i}$ than under $P_{0}^{i}$. Hence, by an application of Lemma $2, l_{q}\left(U_{1}, \cdots, U_{N}\right)$ is stochastically larger under $Q_{0}$ than under $P_{0}$. The first part of the theorem follows. An analogous argument can be used to establish the second part.

The minimax and Neyman-Pearson versions of (P1) can be stated as follows.

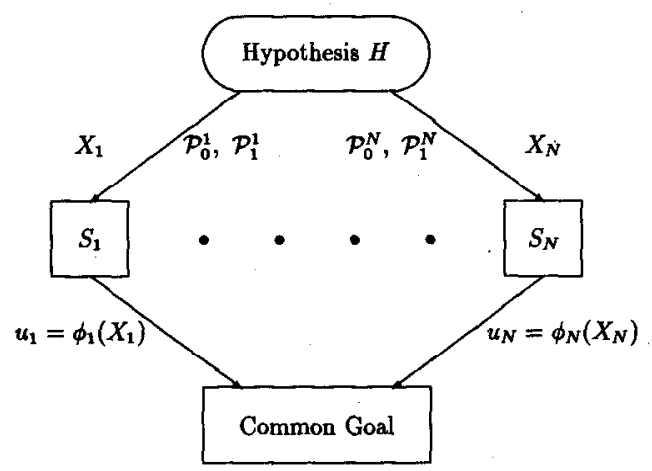

Fig. 2. Robust decentralized detection without a fusion center.

\section{Problem (P2):}

$\inf _{\phi_{1}, \cdots, \phi_{N, \gamma}} \max \left[\sup _{P_{0} \in \mathcal{P}_{0}} P_{F}\left(P_{0}, \phi, \gamma\right), \sup _{P_{1} \in \mathcal{P}_{1}} P_{M}\left(P_{1}, \phi, \gamma\right)\right]$

\section{Problem (P3):}

$\inf _{\phi_{1}, \cdots, \phi_{N}, \gamma} \sup _{P_{1} \in \mathcal{P}_{1}} P_{M}\left(P_{1}, \phi, \gamma\right)$

subject to $\sup _{P_{0} \in \mathcal{P}_{0}} P_{F}\left(P_{0}, \phi, \gamma\right) \leq \alpha$.

Just as in the simple versions of (P2) and (P3), we extend the class of allowable decision functions to include jointly randomized decision rules [3]. In the class of jointly randomized strategies, the simple versions of (P2) and (P3) have solutions that are randomized tests obtained by joint randomization between two deterministic MLRT strategies. Hence, an argument similar to the one given in (1) can be used to show that $\left(Q_{0}, Q_{1}\right)$ are LFD's for (P2) and (P3). The corresponding robust tests are then obtained as solutions to the simple decentralized detection problems in which the sets $\mathcal{P}_{0}$ and $\mathcal{P}_{1}$ are replaced by the singletons $Q_{0}$ and $Q_{1}$, respectively.

\section{ROBUST DECENTRALIZED DETECTION WiThout a Fusion CENTER}

A description of the system under consideration here is shown in Fig. 2, with the only differences from the system in Section III being that the local decisions $U_{i}$ are binary and that there is no fusion center. This setup is useful only in a Bayesian framework in which we assume that the local decisions are coupled through a common cost function $W\left(U_{1}, \cdots, U_{N} ; H\right)$. The expected cost is a function of the conditional distributions at the sensors and the local decision functions. Thus, the expected cost is given by

$$
\begin{aligned}
& C\left(P_{0}, P_{1}, \phi\right)=\nu E_{P_{0}}\{\left.W\left(U_{1}, \cdots, U_{N} ; H_{0}\right)\right\} \\
&+(1-\nu) E_{P_{1}}\left\{W\left(U_{1}, \cdots, U_{N} ; H_{1}\right)\right\}
\end{aligned}
$$

The Bayes minimax robust detection problem at hand is then the following. 


\section{Problem (P4):}

$$
\inf _{\phi} \sup _{\left(P_{0}, P_{1}\right) \in \mathcal{P}_{0} \times \mathcal{P}_{\mathbf{1}}} C\left(P_{0}, P_{1}, \phi\right) .
$$

If the distributions of the observations are known, i.e., if the uncertainty classes $\mathcal{P}_{j}^{i}$ are singletons, then the optimal decision rules for (P4) are binary likelihood ratio tests (LRT's) [3], [9]. Here, we have assumed that for each $i$, the pair $\left(\mathcal{P}_{0}^{i}, \mathcal{P}_{1}^{i}\right)$ is jointly stochastically bounded $\left(Q_{0}^{i}, Q_{1}^{i}\right)$. We showed in Section III that $\left(Q_{0}, Q_{1}\right)$ are LFD's for the Bayesian decentralized detection problem (P1). In the following, we show that $\left(Q_{0}, Q_{1}\right)$ are LFD's for (P4), provided the cost function $W$ satisfies certain conditions.

The distributions $\left(Q_{0}, Q_{1}\right)$ are LFD's for (P4) if for any LRT's $\phi^{R}$ based on $Q_{1}$ and $Q_{0}$, the following inequality holds:

$$
C\left(Q_{0}, Q_{1}, \phi^{R}\right) \geq C\left(P_{0}, P_{1}, \phi^{R}\right), \forall\left(P_{0}, P_{1}\right) \in \mathcal{P}_{0} \times \mathcal{P}_{1}
$$

Now, suppose that $\phi^{*}$ constitutes a solution to the following problem:

$$
\inf _{\phi} C\left(Q_{0}, Q_{1}, \phi\right)
$$

Then, by an argument similar to one following Theorem 1, we can show that $\phi^{*}$ solves (P4).

Of course, any likelihood ratio test $\phi_{i}^{R}$ at sensor $S_{i}$ based on $Q_{1}^{i}$ and $Q_{0}^{i}$ has the form

$$
\phi_{i}^{R}\left(X_{i}\right)= \begin{cases}1 & \text { if } l_{q}^{i}\left(X_{i}\right) \geq t_{i} \\ 0 & \text { if } l_{q}^{2}\left(X_{i}\right)<t_{i}\end{cases}
$$

We now consider some special cases.

\section{A. The Two-Sensor Case}

Here, the expected cost has the form

$$
\begin{aligned}
& C\left(P_{0}, P_{1}, \phi_{1}^{R}, \phi_{2}^{R}\right) \\
& =\nu W\left(0,0 ; H_{0}\right)\left(1-P_{0}^{1}\left(l_{q}^{1}\left(X_{1}\right) \geq t_{1}\right)\right) \\
& \cdot\left(1-P_{0}^{2}\left(l_{q}^{2}\left(X_{2}\right) \geq t_{2}\right)\right) \\
& +\nu W\left(0,1 ; H_{0}\right)\left(1-P_{0}^{1}\left(l_{q}^{1}\left(X_{1}\right) \geq t_{1}\right)\right) \\
& \cdot P_{0}^{2}\left(l_{q}^{2}\left(X_{2}\right) \geq t_{2}\right) \\
& +\nu W\left(1,0 ; H_{0}\right) P_{0}^{1}\left(l_{q}^{1}\left(X_{1}\right) \geq t_{1}\right) \quad \\
& +\nu W\left(1,1 ; H_{0}\right) P_{0}^{1}\left(l_{q}^{1}\left(X_{1}\right) \geq t_{1}\right) \quad \cdot\left(1-P_{0}^{2}\left(l_{q}^{2}\left(X_{2}\right) \geq t_{2}\right)\right) \\
& \quad \cdot P_{0}^{2}\left(l_{q}^{2}\left(X_{2}\right) \geq t_{2}\right)
\end{aligned}
$$$$
+ \text { similar terms in } W\left(i, j ; H_{1}\right) \text {. }
$$

If $W\left(0,0 ; H_{0}\right)=W\left(1,1 ; H_{1}\right)=0$, then for (2) to hold, it is sufficient that the following conditions hold:

$$
W\left(1,1 ; H_{0}\right) \geq W\left(0,1 ; H_{0}\right)+W\left(1,0 ; H_{0}\right)
$$

and

$$
W\left(0,0 ; H_{1}\right) \geq W\left(0,1 ; H_{1}\right)+W\left(1,0 ; H_{1}\right) .
$$

A special case of the above conditions is found in [4] where it is assumed that the cost function is of the form

$$
W\left(U_{1}, U_{2} ; H\right)= \begin{cases}0 & \text { for } U_{1}=U_{2}=H \\ e & \text { for } U_{1} \neq U_{2} \\ f>2 e & \text { for } U_{1}=U_{2} \neq H .\end{cases}
$$

\section{B. The Case of Fixed Symmetric Fusion Rules}

Bayesian decentralized detection problems with binary local decisions and fixed fusion rules such as the "AND" rule and the "OR" rule can be posed in the framework of this section with an appropriately chosen cost function $W$.

For the "AND" rule, a final decision in favor of $H_{1}$ is made whenever all the local decisions are 1; otherwise, a decision in favor of $H_{0}$ is made. If the Bayesian criterion is to minimize the error probability at the fusion center, then the corresponding cost function $W\left(U_{1}, \cdots, U_{N} ; H\right)$ has the form

$W\left(U_{1}, \cdots, U_{N} ; H_{1}\right)= \begin{cases}0 & \text { for } U_{1}=U_{2}=\cdots=U_{N}=1 \\ 1 & \text { otherwise }\end{cases}$

and

$W\left(U_{1}, \cdots, U_{N} ; H_{0}\right)= \begin{cases}1 & \text { for } U_{1}=U_{2}=\cdots=U_{N}=1 \\ 0 & \text { otherwise }\end{cases}$

In this case, the expected cost is

$$
\begin{aligned}
C\left(P_{0}, P_{1}, \phi^{R}\right)=\nu \prod_{i=1}^{N} & P_{0}^{i}\left(l_{q}^{i}\left(X_{i}\right) \geq t_{i}\right) \\
& +(1-\nu)\left(1-\prod_{i=1}^{N} P_{1}^{i}\left(l_{q}^{i}\left(X_{i}\right) \geq t_{i}\right)\right) .
\end{aligned}
$$

A straightforward application of the joint stochastic boundedness property shows that condition (2) holds. That is, $\left(Q_{0}, Q_{1}\right)$ are LFD's for (P4) in this case. A similar result holds for the "OR" fusion rule.

Remark 3: 'The results of this section can be generalized in view of the observation that $\left(Q_{0}, Q_{1}\right)$ are LFD's for (P4) whenever the cost function $W$ satisfies the following monotonicity condition:

$$
\boldsymbol{u} \leq \boldsymbol{v} \Rightarrow \begin{gathered}
W\left(\boldsymbol{u} ; H_{0}\right) \leq W\left(\boldsymbol{v} ; H_{0}\right) \\
\text { and } \\
W\left(\boldsymbol{u} ; H_{1}\right) \geq W\left(\boldsymbol{v} ; H_{1}\right)
\end{gathered}
$$

where, for the vectors $\boldsymbol{u}=\left(u_{1}, \cdots, u_{N}\right)$ and $\boldsymbol{v}=$ $\left(v_{1}, \cdots, v_{N}\right)$, we write $\boldsymbol{u} \leq \boldsymbol{v}$ to mean $u_{i} \leq v_{i}$ for each $i=1, \cdots, N$. Note that this monotonicity condition is satisfied by all of the special cases considered in this section.

\section{CONCLUSION}

We studied decentralized detection problems in which the sensor distributions were not specified completely, i.e., the sensor distributions were assumed to belong to known uncertainty classes. We showed for a broad class of such detection problems that LFD's exist for minimax robust testing between the hypotheses. These LFD's can be obtained by previously known techniques [2], and the corresponding minimax robust tests are then obtained as solutions to simple decentralized 
detection problems for which the sensor distributions are specified to be the LFD's.

We note that the analysis presented in this paper was restricted to static or block detection schemes. Robustification of decentralized sequential detection schemes such as those discussed in [10], [11] remains an interesting problem for future research.

\section{APPENDIX}

Proof of Lemma 2: This lemma follows straightforwardly from Lemma 1 of $[12$, p. 73]. The details are included here for completeness.

Let $L_{1}=\log Z_{i}$. Then it is easy to see that

$$
\mathcal{F}_{i}\left(L_{i}>t_{i}\right)>\mathcal{G}_{i}\left(L_{i}>t_{i}\right), \quad \text { for all } t_{i} \in \mathbb{R} \text {. }
$$

To establish the lemma, we need to show that

$$
\mathcal{F}\left(\sum_{i=1}^{n} L_{i}>t\right) \geq \mathcal{G}\left(\sum_{i=1}^{n} L_{i}>t\right), \quad \text { for all } t \in \mathbb{R} \text {. }
$$

Let $F_{i}(\cdot)$ and $G_{i}(\cdot)$ denote marginal distribution functions of $L_{i}$ under $\mathcal{F}_{i}$ and $\mathcal{G}_{i}$, respectively. Then

$$
F_{i}(x) \leq G_{i}(x), \quad \text { for all } x \in \mathbb{R} .
$$

Now, by [12, Lemma 1, p. 73] there exist independent random variables $V_{1}, \cdots, V_{n}$ and nondecreasing functions $f_{i}(\cdot)$ and $g_{i}(\cdot)$ such that $f_{i}\left(V_{i}\right)$ has distribution function $F_{i}(\cdot), g_{i}\left(V_{i}\right)$ has distribution function $G_{i}(\cdot)$ and $g_{i}(v) \leq f_{i}(v)$ for all $v$. Hence,

$$
\sum_{i=1}^{n} g_{i}\left(V_{i}\right) \leq \sum_{i=1}^{n} f_{i}\left(V_{i}\right)
$$

which implies that the sum $\sum_{i=1}^{n} L_{i}$ is stochastically larger under measure $\mathcal{F}$ than under measure $\mathcal{G}$.

\section{ACKNOWLEDGMENT}

The authors would like to thank B. Hajek for helpful comments and suggestions. They would also like to thank J. Sadowsky for incisive comments and suggestions (including pointing to a technical error) on an earlier version, which have led to improvements in the manuscript, in particular, as regards Remarks 1 and 3, the proof of Theorem 1, and the observation in the streamlined proof of Lemma 2.

\section{REFERENCES}

[1] V. P. Kuznetsov, "Stable rules for discrimination of hypotheses," Probl. Inform. Transm., vol. 18, pp. 41-51, Jan.-Mar. 1982.

[2] S. A. Kassam and H. V. Poor, "Robust techniques for signal processing: A survcy," Proc. IEEE, vol. 73, pp. 433-481, Mar. 1985.

[3] J. N Tsitsiklis, "Decentralized detection," in Advances in Statistical Signal Processing, Vol. 2-Signal Detection, H. V. Poor and J. B. Thomas, Eds. Greenwich, CT: JAI Press, 1990.

[4] E. Geraniotis, "Robust distributed discrete-time block and sequential detection," in Proc. 1987 Conf. Inform. Sci. Syst., Johns Hopkins Univ., Baltimore, MD, Mar. 1987, pp. 354-360.

[5] E. Geraniotis and Y. A. Chau, "On minimax robust data fusion," in Proc. 1988 Conf. Inform. Sci. Syst., Princeton Univ., Princeton, NJ, Mar. 1988, pp. $876-881$

[6] - "Robust data fusion for multisensor detection systems," IEEE Trans. Inform. Theory, vol. 36, pp. 1265-1279, Nov. 1990

[7] P. J. Huber, "A robust version of the probability ratio test," Ann. Math Statist., vol. 36 , pp. 1753-1758, 1965.

[8] P. J. Huber and V. Strassen, "Minimax tests and the Neyman Pearson lemma for capacities," Ann. Statist., vol. 1, no. 2, pp. 251-263, 1973.

[9] R. R. Tenney and N. R. Sandell, Jr., "Detection with distributed sensors," IEEE Trans. Aerosp. Electron. Syst., vol. AES-17, pp. 501-510, July 1981.

[10] V. V. Veeravalli, "Topics in decentralized detection," Ph.D. dissertation, Univ. llinois, Urbana-Champaign, 1992.

[11] V. V. Veeravalli, T. Başar, and H. V. Poor, "Decentralized sequential detection with a fusion center performing the sequential test," IEEE Trans. Inform. Theory, Mar. 1993.

[12] E. L. Lehmann, Testing Statistical Hypotheses. New York: Wiley, 1959 